\title{
Validation of SNP markers for fruit quality and disease resistance loci in apple (Malus $\times$ domestica Borkh.) using the OpenArray ${ }^{\circledR}$ platform
}

\author{
David Chagné 1 , Stijn Vanderzande², Chris Kirk' , Natalie Profitt ${ }^{3}$, Rosemary Weskett ${ }^{3}$, Susan E. Gardiner ${ }^{1}$, \\ Cameron P. Peace ${ }^{2}$, Richard K. Volz $z^{3}$ and Nahla V. Bassil ${ }^{4}$
}

\begin{abstract}
Genome mapping has promised much to tree fruit breeding during the last 10 years. Nevertheless, one of the greatest challenges remaining to tree fruit geneticists is the translation of trait loci and whole genome sequences into diagnostic genetic markers that are efficient and cost-effective for use by breeders, who must select genetically optimal parents and subsequently select genetically superior individuals among their progeny. To take this translational step, we designed the apple International RosBREED SNP Consortium OpenArray v1.0 (IRSCOA v1.0) assay using a set of 128 apple single nucleotide polymorphisms (SNPs) linked to fruit quality and pest and disease resistance trait loci. The Thermo Fisher Scientific OpenArray ${ }^{\circledR}$ technology enables multiplexed screening of SNP markers using a real-time PCR instrument with fluorescent probe-based Taqman ${ }^{\circledR}$ assays. We validated the apple IRSCOA v1.0 multitrait assay by screening 240 phenotyped individuals from the Plant \& Food Research apple cultivar breeding programme. This set of individuals comprised commercial and heritage cultivars, elite selections, and families segregating for traits of importance to breeders. In total, 33 SNP markers of the IRSCOA v1.0 were validated for use in marker-assisted selection (MAS) for the scab resistances Rvi2Nh2, Rvi4Nh4, Rvi6 Nf, fire blight resistance MR5/RLP1, powdery mildew resistance $P / 2$, fruit firmness, skin colour, flavour intensity, and acidity. The availability of this set of validated trait-associated SNP markers, which can be used individually on multiple genotyping platforms available to various apple breeding programmes or re-designed using the flanking sequences, represents a large translational genetics step from genomics to crop improvement of apple.
\end{abstract}

\section{Introduction}

Since the whole genome sequencing of apple ${ }^{1}$, tree fruit biologists worldwide have embraced the powerful promises of genomics. In the last decade, the genomes of further tree fruit species have been fully sequenced ${ }^{2-5}$ and 3285 and 579 quantitative trait loci (QTL) have been identified in the Rosaceae family and in Citrus,

\footnotetext{
Correspondence: David Chagné (David.Chagne@plantandfood.co.nz) ${ }^{1}$ The New Zealand Institute for Plant \& Food Research Ltd (Plant \& Food Research), Palmerston North Research Centre, Palmerston North, New Zealand ${ }^{2}$ Department of Horticulture, Washington State University, Pullman, WA, USA Full list of author information is available at the end of the article.
}

respectively, as of March 2018 [https://www.rosaceae.org/ search/qtl; https://www.citrusgenomedb.org/search/qtl ${ }^{6}$ ]. Despite the democratization of genomics, many argue that it has thus far under-delivered in its promises for enhancing development of superior new tree fruit cultivars. Peace ${ }^{7}$ attributes this slow uptake to a "chasm" that is only gradually being bridged between genomics research and tree fruit breeding: research outcomes often provide genomic knowledge without translating it into useful tools for breeding to support selection decisions. One class of tool that remains compelling is sets of reproducible, helpfully predictive, cost-effective, and 
validated genetic markers that can be readily implemented for marker-assisted selection (MAS) and DNA-informed breeding in general $^{7-9}$.

The pre-requisite for application of MAS is identification of genomic regions associated with a trait of interest, typically via segregation analysis, quantitative trail locus (QTL) analysis, or genome-wide association studies (GWAS). Over the last 20 years, hundreds of trait locusmapping studies for a range of key horticultural characters in tree fruit crops have been published. It is well accepted that MAS functions more reliably for monogenic and oligogenic traits controlled by a few QTLs with medium to large effects (i.e., phenotypic variation for which individual loci explain greater than about $10 \%$ of the variance). Polygenic traits (i.e., phenotypic variation influenced by many loci each with small or minute effect) might be more appropriately addressed using genomewide selection ${ }^{10-12}$, which is not the focus of this study. Many traits of breeding importance in apple are monogenic or oligogenic; nevertheless, the discovery of a QTL is not sufficient for immediate use in MAS and further validation of markers linked to trait loci is required ${ }^{8}$.

Once genetic markers that are associated with trait loci of interest to breeders have been identified, their predictiveness should be validated before they can be applied routinely in MAS. In the context of our study, a marker is validated when it can be implemented routinely in a breeding programme for a specific trait without further testing. Key aspects of validation are that the marker's alleles must be in strong linkage disequilibrium with alleles of interest of the trait locus and that quantified positive or negative effects are consistently assigned to the same marker alleles. Such linkage must be demonstrated in parents of a programme's breeding material. Ideally, the causative mutation for specific phenotypic contrasts should be targeted in marker development, as it represents the ultimate validated genetic assay; however, this physiological connection has rarely been achieved for tree fruit crop traits. Alternatively, the markers should be located close in the genome to candidate genes, which means that fine gene mapping coupled with functional genomics is useful for developing reliable markers for MAS.

Several types of genetic marker technologies are suitable for use in MAS. Quantitative PCR (qPCR) is the gold standard method for reproducible and cost-effective genotyping for human diagnostics ${ }^{13}$, food safety ${ }^{14}$, and agricultural genetics ${ }^{15}$. Such probe-based assays are attractive because they work with small amounts of DNA and can target a specific single nucleotide polymorphism (SNP). Importantly, every molecular biology laboratory is likely to have access to real-time PCR equipment, which means that qPCR-based assays are likely to be adopted widely for MAS. Last but not least, the cost for
qPCR continues to drop, making it cost-effective to use for MAS.

Apple cultivar breeding is a slow and costly process because of the long juvenile period, two to three years' plant propagation period and large plant size, and the application of MAS has been proposed to improve breeding efficiency for apple ${ }^{7,9}$. However, few examples of the application of marker information for selection in apple breeding programmes have been documented to date $^{8}$. An example of the systematic application of such markers is provided by the rootstock breeding programme at Plant \& Food Research (PFR) which annually applies high-throughput MAS for a range of traits, including fire blight resistance from Malus $\times$ robusta 'Robusta 5', woolly apple aphid resistance from several sources, as well as the critical dwarfing trait ${ }^{16,17}$. Chagné et al. ${ }^{26}$ demonstrated the development of a marker for red skin colouration in apple and Baumgartner and colleagues developed a set of SNP markers for fruit quality and disease resistance ${ }^{18}$. MAS has been a routine operation in the Washington State University apple breeding program since 2008 for parents and 2010 for seedlings, targeting several fruit quality traits (Edge-Garza et al. 2010; Evans and Peace ${ }^{8}$ ) and several other U.S. apple breeding programmes are now routinely conducting MAS (J. Luby and S. Brown, pers. comm.). However, the conversion of more QTLs into trait-predictive DNA-based marker assays remains a bottleneck, as does their availability on multiple genotyping platforms ${ }^{7,19}$, both of which must be resolved for MAS to be implemented in any and every apple breeding programme worldwide.

The first objective of the present study was to convert a comprehensive set of 128 SNPs associated with a range of traits targeted in apple breeding into Taqman $^{\circledR}$ qPCR assays using the OpenArray ${ }^{\circledR}$ technology (Thermo Fisher Scientific, Waltham, MA, USA). The second objective was to validate trait predictions by screening the resulting International RosBREED SNP Consortium OpenArray v1.0 assay (IRSCOA v1.0) with DNA from a PFR breeding programme population.

\section{Methods \\ SNP choice for inclusion in array}

A set of 128 SNPs was chosen that included SNPs reported to be associated with 28 trait $\operatorname{loci}^{20-40}$. The targeted traits were those of importance to consumers (fruit firmness, overcolour, maturity time, flavour, and phytochemical composition) or growers (resistances to fire blight, scab, powdery mildew, and woolly apple aphid) (Table 1; Supplemental Table 1). The number of SNPs chosen per locus ranged from one to 11 . All 128 were true SNPs previously evaluated successfully using in vitro technologies such as the Illumina Infinium array ${ }^{22,24}$, genotyping by sequencing ${ }^{21}$, and direct re-sequencing ${ }^{32,33}$. 
Table 1 Description of the 128 single nucleotide polymorphisms (SNPs) chosen for the IRSCOA v1.0 array

\begin{tabular}{|c|c|c|c|c|c|c|}
\hline Trait & $\begin{array}{l}\text { Gene/locus } \\
\text { name }\end{array}$ & LG & SNP ID & $\begin{array}{l}\text { Taqman assay } \\
\text { ID }\end{array}$ & $\begin{array}{l}\text { SNP } \\
\text { type }\end{array}$ & Reference \\
\hline Acidity & Acidity-LG8 & 8 & ss475879081 & AH1SD3T & $\mathrm{G} / \mathrm{T}$ & Kumar et al. ${ }^{34}$ \\
\hline Acidity & Acidity-LG8 & 8 & ss475879082 & $\mathrm{AH} 21 \mathrm{~B} 91$ & $\mathrm{~A} / \mathrm{C}$ & Peace, unpublished \\
\hline Acidity & Acidity-LG8 & 8 & ss475879097 & AH4AAF9 & $\mathrm{C} / \mathrm{T}$ & Peace, unpublished \\
\hline Acidity & Acidity-LG8 & 8 & ss475882878 & $\mathrm{AH} 518 \mathrm{MH}$ & $C / T$ & Peace, unpublished \\
\hline Acidity & Acidity-LG8 & 8 & ss475882879 & AH6R6SP & $\mathrm{T} / \mathrm{G}$ & Kumar et al. ${ }^{34}$ \\
\hline Acidity & Acidity-LG8 & 8 & ss475882882 & AH704YX & $\mathrm{C} / \mathrm{T}$ & Kumar et al. ${ }^{34}$ \\
\hline Acidity & Acidity-LG8 & 8 & ss475882883 & AH89245 & $\mathrm{C} / \mathrm{T}$ & Kumar et al. ${ }^{34}$ \\
\hline Acidity & Acidity-LG8 & 8 & ss475883287 & AHABIAY & $\mathrm{G} / \mathrm{T}$ & Kumar et al. ${ }^{34}$ \\
\hline Acidity & Acidity-LG8 & 8 & ss475883549 & AHBKGG6 & $\mathrm{C} / \mathrm{T}$ & Kumar et al. ${ }^{34}$ \\
\hline Acidity & Mal & 16 & Ma1-SNP1455 & AHCTENE & $A / G$ & Bai et al..$^{20}$ \\
\hline Acidity & Mal & 16 & ss475876558 & AHD2CTM & $\mathrm{A} / \mathrm{C}$ & Chagne, unpublished \\
\hline Acidity/Bitter pit/cracking & LAR1 & 16 & ss475881696 & AHFBAZU & $A / G$ & Kumar et al. ${ }^{34}$ \\
\hline Acidity/Bitter pit/cracking & $L A R 1$ & 16 & ss475881707 & AHGJ852 & $A / G$ & Kumar et al. ${ }^{34}$ \\
\hline Acidity/Bitter pit/cracking & LAR1 & 16 & ss475882555 & AHHS7CA & $C / T$ & Kumar et al. ${ }^{34}$ \\
\hline Acidity/Bitter pit/cracking & LART & 16 & ss475883754 & AHI15II & $C / T$ & Kumar et al. ${ }^{34}$ \\
\hline Acidity/Bitter pit/cracking & LAR1 & 16 & ss475883942 & AHKA3OQ & $\mathrm{A} / \mathrm{C}$ & Kumar et al. ${ }^{34}$ \\
\hline Acidity/Bitter pit/cracking & LAR1 & 16 & ss475884060 & AHLJ1UY & $\mathrm{C} / \mathrm{T}$ & Kumar et al. ${ }^{34}$ \\
\hline $\begin{array}{l}\text { Acidity/Bitter pit/cracking/ } \\
\text { crispness }\end{array}$ & LAR1 & 16 & ss475881704 & AHMSZ06 & $C / T$ & Kumar et al. ${ }^{34}$ \\
\hline Bitter pit & Bp13 & 13 & ss475880861 & AHN1X7E & $\mathrm{A} / \mathrm{C}$ & Peace, unpublished \\
\hline Bitter pit & LAR/Ma & 16 & ss475882553 & AHPAWDM & $\mathrm{G} / \mathrm{T}$ & Peace, unpublished \\
\hline Esters & MdAAT1 & 2 & Fem_cg_22 & AHQJUJU & $\mathrm{T} / \mathrm{C}$ & Dunemann et al. ${ }^{31}$; Bianco et al. ${ }^{22}$ \\
\hline Esters & MdAAT1 & 2 & Fem_cg_23 & AHRSSP2 & $\mathrm{G} / \mathrm{A}$ & Dunemann et al. ${ }^{31}$; Bianco et al. ${ }^{22}$ \\
\hline Esters & MdAAT1 & 2 & Fem_cg_24 & AHS1QWA & $\mathrm{G} / \mathrm{A}$ & Dunemann et al. ${ }^{31}$; Bianco et al. ${ }^{22}$ \\
\hline Esters & MdAAT1 & 2 & ss475876972 & AHUAO2I & $\mathrm{A} / \mathrm{C}$ & Souleyre et al. ${ }^{38}$; Rowan et al. ${ }^{50}$ \\
\hline Esters & MdAAT1 & 2 & ss475876985 & AHVJM8Q & $A / G$ & Souleyre et al. ${ }^{38}$; Rowan et al. ${ }^{50}$ \\
\hline Esters & MdAAT1 & 2 & ss475882633 & AHWSLEY & $A / G$ & Souleyre et al. ${ }^{38}$; Rowan et al. ${ }^{50}$ \\
\hline Ethylene & MdACO1 & 10 & FEM_cg_4 & AHX1JK6 & $A / G$ & Costa et al. ${ }^{30} ;$ Bianco et al. ${ }^{22}$ \\
\hline Ethylene & MdACS1 & 15 & FEM_cg_6 & AHZAHRE & $\mathrm{T} / \mathrm{C}$ & Costa et al. ${ }^{30} ;$ Bianco et al. ${ }^{22}$ \\
\hline Fire blight resistance & $F B E$ & 12 & FBE-1_Y320 & AH704YY & $C / T$ & Jansch et al. ${ }^{33}$ \\
\hline Fire blight resistance & $F B E$ & 12 & FBE-2_Y192 & AH89246 & $C / T$ & Jansch et al. ${ }^{33}$ \\
\hline Fire blight resistance & $F B E$ & 12 & FBE-2_Y495 & AHABIAZ & $C / T$ & Jansch et al. ${ }^{33}$ \\
\hline Fire blight resistance & $F B E$ & 12 & FBE-2_Y551 & AHBKGG7 & $\mathrm{C} / \mathrm{T}$ & Jansch et al. ${ }^{33}$ \\
\hline Fire blight resistance & MR5 & 3 & FB-MR5-NZsnEH034548_K35 & AHOJFXM & $\mathrm{G} / \mathrm{T}$ & Jansch et al. ${ }^{33}$ \\
\hline Fire blight resistance & MR5 & 3 & FB-MR5-NZsnEH034548_R240 & AH1SD3U & $A / G$ & Jansch et al. ${ }^{33}$ \\
\hline Fire blight resistance & MR5 & 3 & FB-MR5-NZsnEH034548_R249 & $\mathrm{AH} 21 \mathrm{~B} 92$ & $A / G$ & Jansch et al. ${ }^{33}$ \\
\hline Fire blight resistance & MR5 & 3 & FB-MR5-rp16k15_M106 & AH4AAGA & $\mathrm{A} / \mathrm{C}$ & Jansch et al. ${ }^{33}$ \\
\hline Fire blight resistance & $R L P 1$ & 3 & RLP1a & $\mathrm{AH} 518 \mathrm{Ml}$ & $\mathrm{C} / \mathrm{A}$ & Gardiner et al. ${ }^{32}$ \\
\hline Fire blight resistance & $R L P 1$ & 3 & RLP1b & AH6R6SQ & $\mathrm{A} / \mathrm{T}$ & Gardiner et al. ${ }^{32}$ \\
\hline
\end{tabular}


Table 1 continued

\begin{tabular}{|c|c|c|c|c|c|c|}
\hline Trait & $\begin{array}{l}\text { Gene/locus } \\
\text { name }\end{array}$ & LG & SNP ID & $\begin{array}{l}\text { Taqman assay } \\
\text { ID }\end{array}$ & $\begin{array}{l}\text { SNP } \\
\text { type }\end{array}$ & Reference \\
\hline Fructose content & LGIFru & 1 & ss475876885 & AHCTENF & $\mathrm{A} / \mathrm{C}$ & Guan et al. ${ }^{39}$ \\
\hline Fructose content & LGIFru & 1 & ss475876892 & AHD2CTN & $\mathrm{A} / \mathrm{G}$ & Guan et al. ${ }^{39}$ \\
\hline Fructose content & LGIFru & 1 & ss475876894 & AHFBAZV & $\mathrm{C} / \mathrm{T}$ & Guan et al. ${ }^{39}$ \\
\hline Fructose content & LGIFru & 1 & ss475882287 & AHGJ853 & $A / G$ & Guan et al..$^{39}$ \\
\hline Fructose content & LGIFru & 1 & ss475883870 & AHHS7CB & $C / T$ & Guan et al. ${ }^{39}$ \\
\hline Fructose content & LGIFru & 1 & ss475884136 & AHI15IJ & $\mathrm{C} / \mathrm{T}$ & Guan et al. ${ }^{39}$ \\
\hline Fruit firmness & LG15-BB & 15 & GDsnp00111 & AHQJUJV & $\mathrm{C} / \mathrm{T}$ & Chagne et al. ${ }^{2,25}$ \\
\hline Fruit firmness & LG15-BB & 15 & GDsnp01763 & AHRSSP3 & $\mathrm{A} / \mathrm{T}$ & Chagne et al. ${ }^{2,25}$ \\
\hline Fruit firmness & LG16-BB & 16 & CONS11 & AHS1QWB & $\mathrm{A} / \mathrm{T}$ & Chagne et al. ${ }^{2,25}$ \\
\hline Fruit firmness & LG16-BB & 16 & GDsnp00071 & AHUAO2J & $\mathrm{A} / \mathrm{T}$ & Chagne et al. ${ }^{2,25}$ \\
\hline Fruit firmness & LG16-BB & 16 & S16_M6073277 & AHVJM8R & $\mathrm{A} / \mathrm{C}$ & Chagne et al. ${ }^{2,25}$ \\
\hline Fruit firmness & LG16-BB & 16 & S16_M6504570 & AHWSLEZ & $\mathrm{A} / \mathrm{C}$ & Chagne et al. ${ }^{2,25}$ \\
\hline Fruit firmness & LG16-BB & 16 & S16_Y5000318 & $\mathrm{AHX} 1 \mathrm{JK} 7$ & $C / T$ & Chagne et al. ${ }^{2,25}$ \\
\hline Fruit firmness & LG16-BB & 16 & S16_Y5639535 & AHZAHRF & $C / T$ & Chagne et al. ${ }^{2,25}$ \\
\hline Fruit firmness & $M d P G 1$ & 10 & FEM_cg_19 & AHKA3OR & $\mathrm{C} / \mathrm{T}$ & Costa et al. ${ }^{29}$; Bianco et al. ${ }^{22}$ \\
\hline Fruit firmness & $M d P G 1$ & 10 & GDsnp02179 & AHLJ1UZ & $T / G$ & Chagne et al. 2,25 \\
\hline Fruit firmness & $M d P G 1$ & 10 & ss475882314 & AHMSZO7 & $A / G$ & Chagne et al. ${ }^{2,25}$ \\
\hline Fruit firmness & $M d P G 1$ & 10 & ss475882315 & AHN1X7F & $\mathrm{A} / \mathrm{C}$ & Chagne et al. ${ }^{2,25}$ \\
\hline Fruit firmness & $M d P G 1$ & 10 & ss475883584 & AHPAWDN & $T / G$ & Chagne et al. ${ }^{2,25}$ \\
\hline Maturity time & Maturity-LG3 & 3 & ss475877739 & AHOJFXN & $\mathrm{G} / \mathrm{T}$ & Peace, unpublished \\
\hline Maturity time & Maturity-LG3 & 3 & ss475877767 & AH1SD3V & $\mathrm{G} / \mathrm{T}$ & Peace, unpublished \\
\hline Maturity time & Maturity-LG3 & 3 & ss475877783 & $\mathrm{AH} 21 \mathrm{~B} 93$ & $C / T$ & Peace, unpublished \\
\hline Maturity time & Maturity-LG3 & 3 & ss475882720 & AH4AAGB & $\mathrm{A} / \mathrm{G}$ & Peace, unpublished \\
\hline Maturity time & Maturity-LG3 & 3 & ss475884066 & AH5l8MJ & $C / T$ & Peace, unpublished \\
\hline Polyphenols & LAR1 & 16 & LAR1_DCk & AH6R6SR & $\mathrm{G} / \mathrm{T}$ & Chagne et al. ${ }^{27}$ \\
\hline Polyphenols & LAR1 & 16 & LAR1_DCr & AH704YZ & $A / G$ & Chagne et al. ${ }^{27}$ \\
\hline Polyphenols & LAR1 & 16 & LAR1_DCy & AH89247 & $C / T$ & Chagne et al. ${ }^{27}$ \\
\hline Polyphenols + Bitter Pit & LAR1 & 16 & ss475881697 & AHABIAO & $C / T$ & Chagne et al. ${ }^{27}$ \\
\hline Polyphenols + Bitter Pit & LAR1 & 16 & ss475883359 & AHBKGG8 & $\mathrm{T} / \mathrm{C}$ & Chagne et al. ${ }^{27}$ \\
\hline Powdery mildew resistance & $P / 2$ & 11 & PI2_3_Y211 & AHCTENG & $A / G$ & Jansch et al. ${ }^{33}$ \\
\hline Powdery mildew resistance & $P / 2$ & 11 & PI2-1_R531 & AHD2CTO & $A / G$ & Jansch et al. ${ }^{33}$ \\
\hline Powdery mildew resistance & $P / 2$ & 11 & PI2-1_Y245 & AHFBAZW & $C / T$ & Jansch et al..$^{33}$ \\
\hline Powdery mildew resistance & Pl2 & 11 & Pl2-1_Y48 & AHGJ854 & $C / T$ & Jansch et al..$^{33}$ \\
\hline Red skin colour & MYB10 & 9 & SNP_FB_0816035 & AHHS7CC & $A / G$ & Chagne et al. ${ }^{26}$ \\
\hline Red skin colour & MYB10 & 9 & SNP_FB_0817523 & AHI15IK & $A / C$ & Chagne et al. ${ }^{26}$ \\
\hline Red skin colour & MYB10 & 9 & SNP_FB_0824598 & AHKA3OS & $A / G$ & Chagne et al. ${ }^{26}$ \\
\hline Red skin colour & MYB10 & 9 & ss475879526 & AHLIU0 & $\mathrm{T} / \mathrm{C}$ & Chagne et al. ${ }^{26}$ \\
\hline Red skin colour & MYB10 & 9 & ss475879531 & AHMSZ08 & $\mathrm{T} / \mathrm{C}$ & Chagne et al. ${ }^{26}$ \\
\hline
\end{tabular}


Table 1 continued

\begin{tabular}{|c|c|c|c|c|c|c|}
\hline Trait & $\begin{array}{l}\text { Gene/locus } \\
\text { name }\end{array}$ & LG & SNP ID & $\begin{array}{l}\text { Taqman assay } \\
\text { ID }\end{array}$ & $\begin{array}{l}\text { SNP } \\
\text { type }\end{array}$ & Reference \\
\hline Red skin colour & MYB10 & 9 & ss475879540 & AHN1X7G & $\mathrm{T} / \mathrm{C}$ & Chagne et al. ${ }^{26}$ \\
\hline Red skin colour & MYB10 & 9 & ss475879551 & AHPAWDO & $A / G$ & Chagne et al. ${ }^{26}$ \\
\hline Red skin colour & MYB10 & 9 & ss475879559 & AHQJUJW & $\mathrm{G} / \mathrm{T}$ & Chagne et al. ${ }^{26}$ \\
\hline Red skin colour & MYB10 & 9 & ss475879574 & AHRSSP4 & $A / G$ & Chagne et al. ${ }^{26}$ \\
\hline Red skin colour & MYB10 & 9 & ss475882942 & AHS1QWC & $A / G$ & Chagne et al. ${ }^{26}$ \\
\hline Red skin colour & MYB10 & 9 & ss475883301 & AHUAO2K & $A / G$ & Chagne et al. ${ }^{26}$ \\
\hline Scab resistance & Rvil1 & 2 & Rvi11-1_Y111 & AHOJFXO & $\mathrm{C} / \mathrm{T}$ & Jansch et al..$^{33}$ \\
\hline Scab resistance & Rvil1 & 2 & Rvi11-1_Y60 & AH1SD3W & $C / T$ & Jansch et al. ${ }^{33}$ \\
\hline Scab resistance & Rvil1 & 2 & Rvi11-2_R357 & $\mathrm{AH} 21 \mathrm{~B} 94$ & $A / G$ & Jansch et al. ${ }^{33}$ \\
\hline Scab resistance & Rvi11 & 2 & Rvi11-2_R60 & AH4AAGC & $A / G$ & Jansch et al. ${ }^{33}$ \\
\hline Scab resistance & Rvil1 & 2 & Rvi11-2_Y733 & AH5I8MK & $C / T$ & Jansch et al. ${ }^{33}$ \\
\hline Scab resistance & Rvil2 & 12 & SNP_23.31_297Y & AHOJFXP & $\mathrm{T} / \mathrm{C}$ & Padmarasu et al. ${ }^{37}$ \\
\hline Scab resistance & Rvi12 & 12 & SNP_23.488_173Y & AH1SD3X & $\mathrm{T} / \mathrm{C}$ & Padmarasu et al. ${ }^{37}$ \\
\hline Scab resistance & Rvil2 & 12 & SNP_24.78_421M & AH21B95 & $\mathrm{A} / \mathrm{C}$ & Padmarasu et al. ${ }^{37}$ \\
\hline Scab resistance & Rvi12 & 12 & SNP_24.85_275W & AH4AAGD & $\mathrm{A} / \mathrm{T}$ & Padmarasu et al. ${ }^{37}$ \\
\hline Scab resistance & Rvil2 & 12 & SNP_23.31_102Y & AHZAHRH & $\mathrm{T} / \mathrm{C}$ & Padmarasu et al. ${ }^{37}$ \\
\hline Scab resistance & Rvi15Nr2 & 2 & Rvi15-1_M75 & AH6R6SS & $\mathrm{A} / \mathrm{C}$ & Jansch et al. ${ }^{33}$ \\
\hline Scab resistance & Rvi15Nr2 & 2 & Rvi15-1_S188 & AH704YO & $\mathrm{G} / \mathrm{C}$ & Jansch et al. ${ }^{33}$ \\
\hline Scab resistance & Rvi15Nr2 & 2 & Rvi15-1_S49 & AH89248 & $\mathrm{G} / \mathrm{C}$ & Jansch et al. ${ }^{33}$ \\
\hline Scab resistance & Rvi15Nr2 & 2 & Rvi15-9C10T7_M264 & AHABIA1 & $\mathrm{A} / \mathrm{C}$ & Jansch et al. ${ }^{33}$ \\
\hline Scab resistance & Rvi15Nr2 & 2 & Rvi15-9C10T7_S296 & AHBKGG9 & $\mathrm{G} / \mathrm{C}$ & Jansch et al..$^{33}$ \\
\hline Scab resistance & Rvi15/Nr2 & 2 & Rvi15-9C10T7_Y224 & AHCTENH & $C / T$ & Jansch et al. ${ }^{33}$ \\
\hline Scab resistance & Rvi2 & 2 & Rvi2_region53_M417 & AHD2CTP & $\mathrm{G} / \mathrm{T}$ & Jansch et al. ${ }^{33}$ \\
\hline Scab resistance & Rvi2 & 2 & Rvi2-1_R239 & AHFBAZX & $A / G$ & Jansch et al. ${ }^{33}$ \\
\hline Scab resistance & Rvi2 & 2 & Rvi2-4_R531 & AHGJ855 & $A / G$ & Jansch et al. ${ }^{33}$ \\
\hline Scab resistance & Rvi2 & 2 & Rvi2-6_R332 & AHHS7CD & $A / G$ & Jansch et al..$^{33}$ \\
\hline Scab resistance & Rvi2 & 2 & Rvi2-7_W242 & AHI15IL & $\mathrm{A} / \mathrm{T}$ & Jansch et al..$^{33}$ \\
\hline Scab resistance & Rvi2 & 2 & Rvi2-8_M417 & АНКАЗОТ & $\mathrm{A} / \mathrm{C}$ & Jansch et al. ${ }^{33}$ \\
\hline Scab resistance & Rvi3 & 4 & S4_K22736301 & AHPAWDP & $\mathrm{T} / \mathrm{G}$ & Bastiaanse et al. ${ }^{21}$ \\
\hline Scab resistance & Rvi3 & 4 & S4_M24705633 & AHQJUJX & $\mathrm{A} / \mathrm{C}$ & Bastiaanse et al. ${ }^{21}$ \\
\hline Scab resistance & Rvi3 & 4 & S4_R22444934 & AHRSSP5 & $A / G$ & Bastiaanse et al. ${ }^{21}$ \\
\hline Scab resistance & Rvi3 & 4 & S4_R22535545 & AHS1QWD & $A / G$ & Bastiaanse et al. ${ }^{21}$ \\
\hline Scab resistance & Rvi3 & 4 & S4_R22561770 & AHUAO2L & $A / G$ & Bastiaanse et al. ${ }^{21}$ \\
\hline Scab resistance & Rvi3 & 4 & S4_R22721144 & AHVJM8T & $A / G$ & Bastiaanse et al. ${ }^{21}$ \\
\hline Scab resistance & Rvi3 & 4 & S4_Y22684914 & AHWSLE1 & $C / T$ & Bastiaanse et al. ${ }^{21}$ \\
\hline Scab resistance & Rvi3 & 4 & S4_Y24706726 & AHX1JK9 & $\mathrm{C} / \mathrm{T}$ & Bastiaanse et al. ${ }^{21}$ \\
\hline Scab resistance & Rvi4 & 2 & Rvi4-1_K146 & AHL1U1 & $\mathrm{T} / \mathrm{G}$ & Jansch et al. ${ }^{33}$ \\
\hline Scab resistance & Rvi4 & 2 & TNL1_R131 & AHMSZ09 & $A / G$ & Jansch et al..$^{33}$ \\
\hline
\end{tabular}


Table 1 continued

\begin{tabular}{|c|c|c|c|c|c|c|}
\hline Trait & $\begin{array}{l}\text { Gene/locus } \\
\text { name }\end{array}$ & LG & SNP ID & $\begin{array}{l}\text { Taqman assay } \\
\text { ID }\end{array}$ & $\begin{array}{l}\text { SNP } \\
\text { type }\end{array}$ & Reference \\
\hline Scab resistance & Rvi4 & 2 & TNL1_R202 & AHN1X7H & $C / T$ & Jansch et al. ${ }^{33}$ \\
\hline Scab resistance & Rvi6 & 1 & M18_Y32 & AHVJM8S & $\mathrm{C} / \mathrm{T}$ & Jansch et al. ${ }^{33}$ \\
\hline Scab resistance & Rvi6 & 1 & M8S_R156 & AHWSLEO & $A / G$ & Jansch et al. ${ }^{33}$ \\
\hline Scab resistance & Rvi6 & 1 & M8S_R193 & $\mathrm{AHX1JK8}$ & $A / G$ & Jansch et al. ${ }^{33}$ \\
\hline Scab resistance & Rvi6 & 1 & Rvi6_42M10SP6_Y124 & AHZAHRG & $\mathrm{C} / \mathrm{T}$ & Jansch et al. ${ }^{33}$ \\
\hline Type 2 red flesh & MYB110 & 17 & S17_R24968227 & $\mathrm{AH} 518 \mathrm{ML}$ & $A / G$ & Chagne et al. ${ }^{28}$ \\
\hline Type 2 red flesh & MYB110 & 17 & S17_Y24974945 & AH6R6ST & $C / T$ & Chagne et al. ${ }^{28}$ \\
\hline Type 2 red flesh & MYB110 & 17 & S17_Y24974945C & $\mathrm{AH} 704 \mathrm{Y} 1$ & $C / T$ & Chagne et al..$^{28}$ \\
\hline Type 2 red flesh & MYB110 & 17 & S17_Y24974945t & AH89249 & $C / T$ & Chagne et al. ${ }^{28}$ \\
\hline Type 2 red flesh & MYB110 & 17 & S17_Y24974965 & AHABIA2 & $C / T$ & Chagne et al. ${ }^{28}$ \\
\hline Type 2 red flesh & MYB110 & 17 & S17_Y24974992 & AHBKGHA & $\mathrm{G} / \mathrm{T}$ & Chagne et al..$^{28}$ \\
\hline Vitamin C & GGP1 & 11 & MdGGP1_885 & AHD2CTQ & $\mathrm{T} / \mathrm{C}$ & Mellidou et al. ${ }^{36}$ \\
\hline Vitamin C & GGP3 & 10 & MdGGP3_211 & AHCTENI & $\mathrm{T} / \mathrm{C}$ & Mellidou et al. ${ }^{36}$ \\
\hline $\begin{array}{l}\text { Woolly apple aphid } \\
\text { resistance }\end{array}$ & Ermis & 7 & GDsnp01994 & AHFBAZY & $C / T$ & Bus et al. ${ }^{23}$ \\
\hline
\end{tabular}

The target trait(s), locus, or candidate gene, linkage group (LG), published SNP identifier (SNP ID), the Taqman assay SNP assay identifier (assay ID) from Thermo Fisher Scientific, SNP type, and publication where each marker-trait association was described are indicated

\section{Plant material}

For validation of the 128 SNPs, 207 accessions from the PFR apple breeding programme were chosen (Supplemental Table 2a). Forty-one accessions were commercial cultivars, 81 were advanced selections and/or breeding parents from PFR, and 85 were seedlings from small breeding families segregating for fruit quality and for disease resistance/susceptibility (specifically the trait loci of $P l 2, R v i 2 / V h 2$, and $R v i 6 / V f)$. Individual seedlings were from seven validation families (6 to 11 seedlings per family) that were planted in the orchard in 2011, as part of a population used in cultivar breeding at PFR, Hawkes Bay, New Zealand ${ }^{17}$. All but one family had been shown to carry resistance alleles at both Rvi2 and Rvi6 following screening in the glasshouse for Rvi2 (by phenotypic selection for stellate necrotic resistance reactions after Venturia inaequalis inoculations) and then by MAS for Rvi6 (using an unpublished high-resolution melting SNP marker). The $\mathrm{Pl} 2$ phenotype was not determined in the seven validation families. The $P l 2$ phenotype was assessed for advanced selections using an unpublished marker (S. Gardiner, pers. comm.). A further 20 individuals were positive and negative controls from trait mapping populations segregating for fire blight resistance from Malus $\times$ robusta 'Robusta $5^{, 32}$ or scab resistances Rvi4/Vh4 $4^{41}$, $R v i 3 / V h 3^{21}$, and $R v i 11 / V b j$ (V. Bus, pers. comm.).
The data set of phenotypic measurements made on the apple accessions screened with the markers included: backward BLUP values for 56 advanced selections and 41 commercial cultivars, average phenotypic values over multiple years for 77 advanced selections and 15 commercial cultivars, and average phenotype values for 65 seedlings of the validation families. The number of founding cultivars was 47, 43 and 27 for the accessions with backward BLUP values, average phenotypic values over multiple years and validation families, respectively (Supplementary Table $2 \mathrm{~b}$ ), with 40 founders common to the groups with backward BLUP and average phenotypic values. All founders for the validation families were represented in each of the other two groups.

\section{Fruit phenotyping}

Each year, a sample of five to six fruit was harvested from each accession when they were gauged to be mature [based on a change in skin background colour from green to white/green/yellow/pale yellow and/or average starch pattern index score of another two to three fruit on a scale from $0(100 \%$ starch) to 6 (0\% starch)]. Each seedling was sampled once to three times at weekly intervals and fruit were cold stored immediately for ten weeks at $0.5^{\circ} \mathrm{C}$ followed by one week at $20^{\circ} \mathrm{C}$. Fruit quality assessments were carried out by up to four trained PFR apple breeding 
personnel. Fruit flesh texture (firmness and crispness) as well as aroma, acidity, sweetness, and flavour intensity were scored for each sample from 0 (low) to 9 (high) using standard foods as references to anchor key scores. Fruit quality assessments for the validation families were conducted in one or two harvest years on two-year-old to three-year-old seedlings, while for the commercial cultivars and advanced selections assessments were conducted in one to fourteen years. In all cases, data were averaged over years for each accession.

\section{Breeding value calculation}

The breeding value of an individual measures the average effect of the genes that are transmitted from parent to progeny and is a more important measure of a parent's worth than its phenotype ${ }^{42}$. For marker-assisted parental selection therefore, the relationship between marker genotype and breeding value is of particular interest to the breeder. Backwards breeding values were determined for advanced selections and commercial cultivars based on the performance of their progenies in the PFR breeding programme. Firmness and crispness scores were collected from 21,852 individual seedlings in 171 breeding families that had been assessed by the breeding programme from 2001 to 2014. For each seedling, the scores for individual fruit samples were determined using the methods described above and averaged over harvests within a year and over years.

A linear mixed model approach was used to fit the individual plant model $^{43}$, with the general mean as the only fixed effect. Seedling and family were treated as separate random effects, i.e., the plant model with the pedigree information incorporated:

$$
\mathbf{y}=\mathbf{X} \boldsymbol{\beta}+Z_{1} \mathbf{a}+Z_{2} \mathbf{b}+\mathbf{e}
$$

where $\mathbf{y}$ is the vector of observed trait scores, $\mathbf{X}, \mathbf{Z}_{\mathbf{1}}$, and $\mathbf{Z}_{2}$ are the incidence matrices for the fixed (mean only), random additive genetic effects (of individual seedlings), and random non-additive genetic (dominance) effects (of individual families), respectively; $\boldsymbol{\beta}$, $\mathbf{a}$, and $\mathbf{b}$ are the vectors of coefficients for the fixed, random additive genetic, and random non-additive genetic effects, respectively, and $\mathbf{e}$ is the vector of random residuals. The associated variances with the random effects, $a, b$, and e are $\sigma_{a}^{2}, 0.25 \sigma_{d}^{2}$ and $\sigma_{e}^{2}$, respectively.

This model was tested against one that excluded the non-additive genetic effect with the likelihood ratio test used to compare the two models for firmness and crispness scores. Diagnostic plots were satisfactory for both models for both traits; however, inclusion of non-additive effect had no influence on log-likelihoods, and therefore the simpler model sufficed. Variance components for random effects of seedling and residual error were determined for each trait and best linear unbiased predictors (BLUPs) as estimates of breeding values, and their standard errors derived for the random effects, were computed for all seedlings and parents. All statistical analyses and graphs were conducted using $\mathrm{R} 2.13 .0^{44}$, and the mixed models were fitted using the asreml-r package $^{45}$. One-way ANOVA using GenStat version 17 (VSN International) was used to test association between markers and BLUP or phenotypic values. Missing data points recorded as " $x$ " or "NA" due to failed qPCR reactions or missing phenotypic data were not included in the ANOVA.

\section{OpenArray ${ }^{\circledast}$ methodologies}

Total DNA was extracted using a cetyl trimethylammonium bromide-based buffer and re-suspended in TE buffer (10 mM Tris; $0.1 \mathrm{mM}$ EDTA). DNA concentration was quantified with Quant-iT ${ }^{\mathrm{Dx}}$ PicoGreen $^{\circledR}$ (Thermo Fisher Scientific) fluorimetry and normalised to $50 \mathrm{ng} / \mu \mathrm{l}$. Samples were sent to Thermo Fisher Scientific

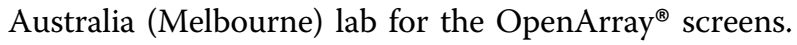

All 128 Taqman $^{\circledast}$ assays were designed using the online Custom Taqman $^{\circledR}$ Assay Design Tool (www. thermofisher.com) and the experiment was established in the 128-plex OpenArray ${ }^{\circledR}$ format.

DNA extracts $(3 \mu \mathrm{L}$ each) were mixed with an equal volume of TaqMan ${ }^{\circledR}$ OpenArray ${ }^{\circledR}$ Genotyping Master Mix using the AccuFill ${ }^{\mathrm{Tm}}$ System for the OpenArray ${ }^{\circledR}$ RealTime PCR Platform prior to analysis on Custom TaqMan ${ }^{\circledast}$ OpenArray ${ }^{\circledR}$ Genotyping Plates using the QuantStudio $^{\text {tw }} 12$ K Flex Real-Time PCR System (Thermo Fisher Scientific). Results were analysed within both the QuantStudio $^{\mathrm{Tm}}$ 12K Flex software and Taqman ${ }^{\circledR}$ Genotyper application (Thermo Fisher Scientific). As the assays were newly designed, each call was manually examined by viewing the real-time trace and the endpoint call. Any manual changes were saved using the Taqman ${ }^{\circledR}$ Genotyper software and exported as a matrix of genotypic calls for each individual sample.

\section{Results \\ IRSCOA v1.0 evaluation}

Of the 128 SNP markers on the IRSCOA v1.0 assay, 110 (85.9\%) resulted in successful PCR amplification and could be scored (Supplemental Table 3 and Supplemental Table 4). The remaining markers had either poor or no PCR amplification and the genotypic calls could not be resolved. The median call rate for the individuals was 94.5\%, calculated as the proportion of SNPs giving a successful genotypic call for each individual, with two individual samples exhibiting a low call rate due to low DNA quality: 'Ralls Janet' (30.9\%) and App1NHCN.01 $(58.2 \%)$. The median call rate for the 110 polymorphic markers was $99 \%$, calculated as the proportion of 
individuals giving a successful genotypic call for each SNP, with only one SNP assay exhibiting a low call rate (AHGJ854; 58\%).

\section{SNP markers for pest and disease resistance}

Of the six SNPs chosen as possible markers for the scab resistance locus Rvi2/Vh2, four were polymorphic (Table 2). More than $40 \%$ of susceptible individuals (thus lacking $R v i 2 / V h 2$ ) displayed a heterozygous genotype for markers AHFBAZX, AHGJ855, and AHHS7CD (Table 2) and so these markers were rejected. However, all individuals heterozygous for AHI15IL (genotype A/T) were expected to carry the Rvi2/Vh2 resistance allele based on their pedigree and phenotyping (Volz and Gardiner, unpublished), while of 149 homozygous $\mathrm{T} / \mathrm{T}$ individuals, 139 were expected to not carry the resistance allele.

Of three assays included in the IRSCOA v1.0 array for the Rvi4/Vh4 resistance locus, all amplified DNA and one (AHLJ1U1) resulted in a marker that was strongly associated with resistance (Table 2): all 204 susceptible and two resistant control individuals exhibited G/G and T/G genotypes, respectively. The two other Rvi4/Vh4 markers were monomorphic (AHMSZ09 and AHN1X7H).

For $R v i 6 / V f$, all four markers included in the IRSCOA v1.0 were polymorphic and strongly associated with resistance (Table 2): all individuals heterozygous for the three markers AHX1JK8, AHVJM8S, and AHWSLE0 were resistant (including Malus floribunda 821 and 'Prima'), except for one individual ('Blenheim Orange'). For marker AHZAHRG, nine individuals not expected to have inherited Rvi6/Vf resistance according to their pedigree instead exhibited a heterozygous (resistant) genotype.

From the eight markers chosen for Rvi3/Vh3, only one was polymorphic; however, it was not associated with resistance (data not shown). Of the five markers chosen for $R v i 11 / V b j$, four were polymorphic, but none was associated with resistance as none was heterozygote for both resistant individuals (ILPE6 and ILPDN) and homozygote for the rest of the individuals.

Five of six markers (AH0JFXM, AH21B92, AH4AAGA, AH5I8MI, and AH6R6SQ) developed for the fire blight resistance derived from Malus x robusta 'Robusta 5' (MR5/RLP1) were polymorphic and successfully distinguished resistant from susceptible phenotypes as two resistant individuals from the 'Malling 9' $\times$ 'Robusta 5' population were heterozygous at the respective SNP positions while the remaining susceptible individuals were homozygous (Table 2).

Three markers out of four were polymorphic for the $P l 2$ powdery mildew resistance (marker AHCTENG was monomorphic). Two Pl2 markers (AHD2CTO and AHFBAZW) co-segregated perfectly with phenotype (Table 2). One accession genotyped with AHD2CTO was
Table 2 Validation of single nucleotide polymorphism (SNP) markers for apple scab, fire blight, and powdery mildew resistance loci using the IRSCOA v1.0 array

\begin{tabular}{lllll}
\hline Locus & Marker & Susceptible & Resistant & Chi-square ( $\boldsymbol{p}$-value) \\
\hline Rvi6Nf & AHX1JK8 & & & \\
& A/A & 125 & 5 & $p<0.00001$ \\
& A/G & 0 & 70 & $p<0.00001$ \\
& G/G & 1 & 3 & 0.317 \\
Rvi6Nf & AHVJM8S & & & \\
& C/C & 124 & 7 & $p<0.00001$ \\
& C/T & 1 & 67 & $p<0.00001$ \\
& T/T & 1 & 4 & 0.179 \\
Rvi6Nf & AHZAHRG & & & \\
& C/C & 117 & 4 & $p<0.00001$ \\
& C/T & 9 & 71 & $p<0.00001$ \\
& T/T & 3 & 3 & 1
\end{tabular}

RVIGNf AHWSLEO

$\begin{array}{llll}\text { G/G } & 127 & 8 & p<0.00001 \\ \mathrm{~A} / \mathrm{G} & 1 & 70 & p<0.00001\end{array}$

Rvi2Nh2 AHI15IL

$\begin{array}{lll}T / T & 139 & 10\end{array}$

$p<0.00001$

$p<0.00001$

Rvi2Nh2 AHFBAZX

A/A $\quad 2$

A/G $\quad 43$

G/G $\quad 82$

0.351

$p<0.00001$

Rvi2Nh2 AHGJ855

G/G $\quad 62$

$\mathrm{A} / \mathrm{G}$

$p<0.00001$

0.039

Rvi2Nh2 AHHS7CD

A/A $\quad 51 \quad 6$

A/G $\quad 74$

0.0001

$\mathrm{G} / \mathrm{G}$

0.205

Rvi4Nh4 AHLJ1U1

G/G 204

T/G 0

$p<0.00001$

0.157

Rvi11Nbj AHOJFXO

$\mathrm{T} / \mathrm{T} \quad 187$

$p<0.00001$

0.0001

Rvi11Nbj AH1SD3W

$\begin{array}{llll}C / C & 181 & 0 & p<0.00001 \\ C / T & 20 & 1 & p<0.00001\end{array}$


Table 2 continued

\begin{tabular}{|c|c|c|c|c|}
\hline Locus & Marker & Susceptible & Resistant & Chi-square ( $p$-value) \\
\hline & $T / T$ & 5 & 1 & 0.102 \\
\hline \multirow[t]{3}{*}{ Rvi11 Nbj } & AH4AAGC & & & \\
\hline & $\mathrm{G} / \mathrm{G}$ & 4 & 0 & $p<0.00001$ \\
\hline & $\mathrm{A} / \mathrm{G}$ & 197 & 2 & $p<0.00001$ \\
\hline \multirow[t]{4}{*}{ Rvi11 Nbj } & AH5I8MK & & & \\
\hline & $\mathrm{C} / \mathrm{C}$ & 193 & 1 & $p<0.00001$ \\
\hline & $C / T$ & 6 & 0 & $p<0.00001$ \\
\hline & $T / T$ & 1 & 0 & \\
\hline \multirow[t]{3}{*}{ MR5/RLP1 } & AHOJFXM & & & \\
\hline & $\mathrm{G} / \mathrm{T}$ & 1 & 2 & \\
\hline & $T / T$ & 202 & 0 & $p<0.00001$ \\
\hline \multirow[t]{3}{*}{ MR5/RLP1 } & $\mathrm{AH} 21 \mathrm{~B} 92$ & & & \\
\hline & $\mathrm{G} / \mathrm{G}$ & 200 & 0 & $p<0.00001$ \\
\hline & $A / G$ & 4 & 2 & 0.414 \\
\hline \multirow[t]{3}{*}{ MR5/RLP1 } & AH4AAGA & & & \\
\hline & $C / C$ & 199 & 0 & $p<0.00001$ \\
\hline & $\mathrm{A} / \mathrm{C}$ & 5 & 2 & 0.256 \\
\hline \multirow[t]{3}{*}{ MR5/RLP1 } & $\mathrm{AH} 518 \mathrm{Ml}$ & & & \\
\hline & $\mathrm{C} / \mathrm{C}$ & 197 & 0 & $p<0.00001$ \\
\hline & $\mathrm{A} / \mathrm{C}$ & 3 & 2 & 0.654 \\
\hline \multirow[t]{3}{*}{ MR5/RLP1 } & AH6R6SQ & & & \\
\hline & A/A & 201 & 0 & $p<0.00001$ \\
\hline & $A / T$ & 1 & 2 & 0.563 \\
\hline \multirow[t]{4}{*}{$P 12$} & AHD2CTO & & & \\
\hline & $\mathrm{A} / \mathrm{A}$ & 88 & 0 & $p<0.00001$ \\
\hline & $A / G$ & 0 & 6 & $p<0.00001$ \\
\hline & $\mathrm{G} / \mathrm{G}$ & 1 & 0 & \\
\hline \multirow[t]{3}{*}{$P 12$} & AHFBAZW & & & \\
\hline & $\mathrm{C} / \mathrm{T}$ & 1 & 6 & 0.037 \\
\hline & $\mathrm{T} / \mathrm{T}$ & 89 & 0 & $p<0.00001$ \\
\hline \multirow[t]{4}{*}{$P / 2$} & AHGJ854 & & & \\
\hline & $\mathrm{C} / \mathrm{C}$ & 10 & 6 & 0.354 \\
\hline & $C / T$ & 3 & 0 & \\
\hline & $\mathrm{T} / \mathrm{T}$ & 70 & 0 & $p<0.00001$ \\
\hline
\end{tabular}

The number of resistant and susceptible individuals for each genotypic class is indicated and the p-value for a chi-square test is given

homozygous resistant G/G and the same individual DNA sample was the only heterozygote observed for marker AHFBAZW.

No positive controls were screened for Rvi12/Vb, Rvi15/

$\mathrm{Vr} 2$, woolly apple aphid Er4 resistance derived from MIS o.p. [open pollinated seedling of 'Mildew Immune Selection' (MIS), itself an open-pollinated selection of 'Delicious'], or fire blight resistance derived from 'Evereste'. Hence, although two, five, one and one assays, respectively, generated polymorphisms from the array, it was not possible to determine their utility for MAS.

\section{Marker validation for fruit crispness and firmness}

All 28 SNP assays for fruit crispness and firmness were scorable and polymorphic. These assays were 15 SNPs across five loci [MdPG1 and MdACO1 on LG10, MdACS1 and QTLs derived from 'Braeburn' on LG15 (LG15-BB), and LG16 (LG16-BB)] and 13 assays around the LAR1 and Ma1 loci on LG16. The data set of phenotypic measurements for the advanced selection, commercial cultivars and validation families is presented in Supplemental Fig. 1.

Significant marker-trait associations were identified for four of the five $M d P G 1$ markers. For $M d P G 1$, the BLUP values indicated that the $C$ allele of AHKA3OR was associated with firmer and crisper fruit (Fig. 1). For the MdPG1 SNPs of AHN1X7F, AHPAWDN, and AHMSZ07, alleles $\mathrm{A}, \mathrm{T}$, and $\mathrm{A}$, respectively, were associated with firmer and crisper fruit (Supplemental Fig. 2). The significant effect of AHKA3OR was confirmed in the validation families for both fruit firmness and crispness, while it could not be confirmed for crispness and firmness $(p>0.05)$ using averaged phenotypic data of the advanced selections and commercial cultivars. Association of AHN1X7F was confirmed with fruit firmness $(p=0.018)$ from phenotypic data of the validation families; however, significant associations were not detected using phenotypic data of the advanced selections and commercial cultivars. Marker AHPAWDN was significantly associated with the BLUPs for crispness $(p=0.032)$, but not with the raw phenotypes. AHMSZ07 was significantly associated with BLUPs for crispness and firmness $(p<0.0001)$ and raw phenotypes in the validation families for crispness $(p=$ 0.009), but not for firmness or in the advanced selections. The single LG10 marker for MdACO1, AHX1JK6, did not explain any of the variation in the BLUPs or raw phenotypes for crispness and firmness in the advanced selections, commercial cultivars, or validation families.

The assay for MdACS1 on LG15, AHZAHRE, gave significant associations for the BLUP values for crispness and firmness of the advanced selections and commercial cultivars but not for the averaged phenotypic values of the same accessions. The $C$ allele was associated with higher BLUP-values for both traits (Fig. 2). At the LG15-BB locus, the $\mathrm{C}$ allele from assay AHQJUJV was associated with the BLUP values for both crispness and firmness (Supplemental Fig. 2), however not significantly $(p=$ 0.065 and $p=0.096$, respectively). 


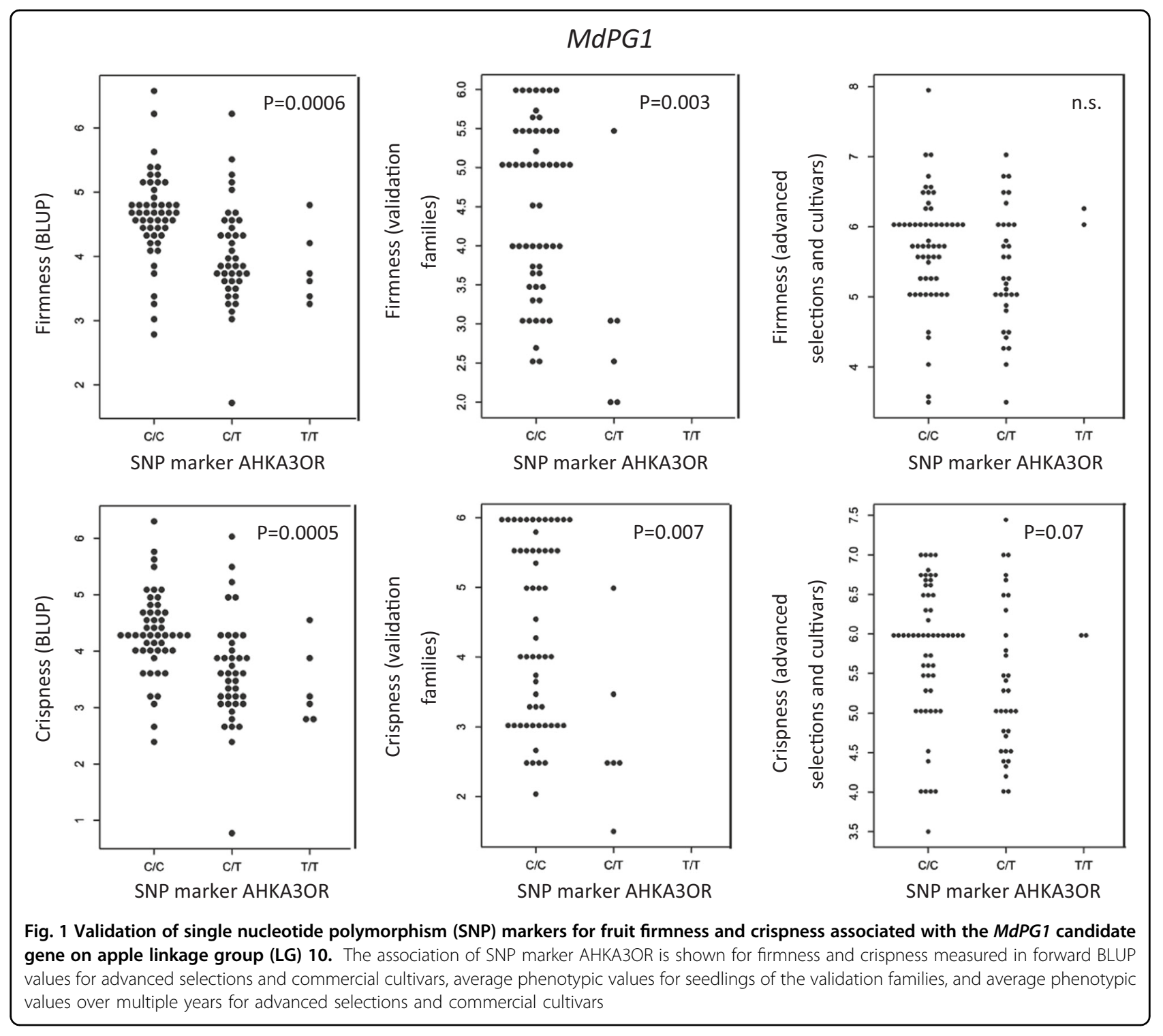

For the LG16-BB locus on LG16, three markers (AHS1QWB, AHZAHRF, and AHUAO2J) of six tested were significantly associated with BLUP values for both crispness and firmness in the advanced selections and commercial cultivars, but their effects were not confirmed using any of the averaged phenotypic data. For AHS1QWB, individuals with the homozygous TT genotype exhibited BLUP values indicating firmer and crisper apples (Fig. 2); however, the TT genotype was associated with lower averaged phenotypic values in the validation families (Supplemental Fig. 2). For AHZAHRF, lower BLUP values for crispness and firmness corresponded to the homozygous TT genotype $(p=0.028$ and $p=0.046$, respectively; Supplemental Fig. 2). Heterozygotes for AHUAO2J were associated with lower BLUP values for both firmness and crispness, while the homozygous genotypes were associated with similar BLUP values. The heterozygote/homozygote difference for AHUAO2J was not detected using the averaged phenotypes. The remaining three markers for LG16-BB (AHWSLEZ, AHX1JK7 and AHVJM8R) were not associated with any of the phenotypes.

Twelve of the 13 Ma1-linked and LAR1-linked markers on LG16 were significantly associated with firmness or crispness or both. The A, A, C, C, and G alleles of AHFBAZU, AHKA3OQ AHLJ1UY, AHI15II, and AH704YZ, respectively (Supplemental Fig. 2), were significantly associated with higher crispness and firmness based on BLUP values in the advanced selections and commercial cultivars. However, these associations were not confirmed using the averaged phenotypes of advanced selections, commercial cultivars, and validation 


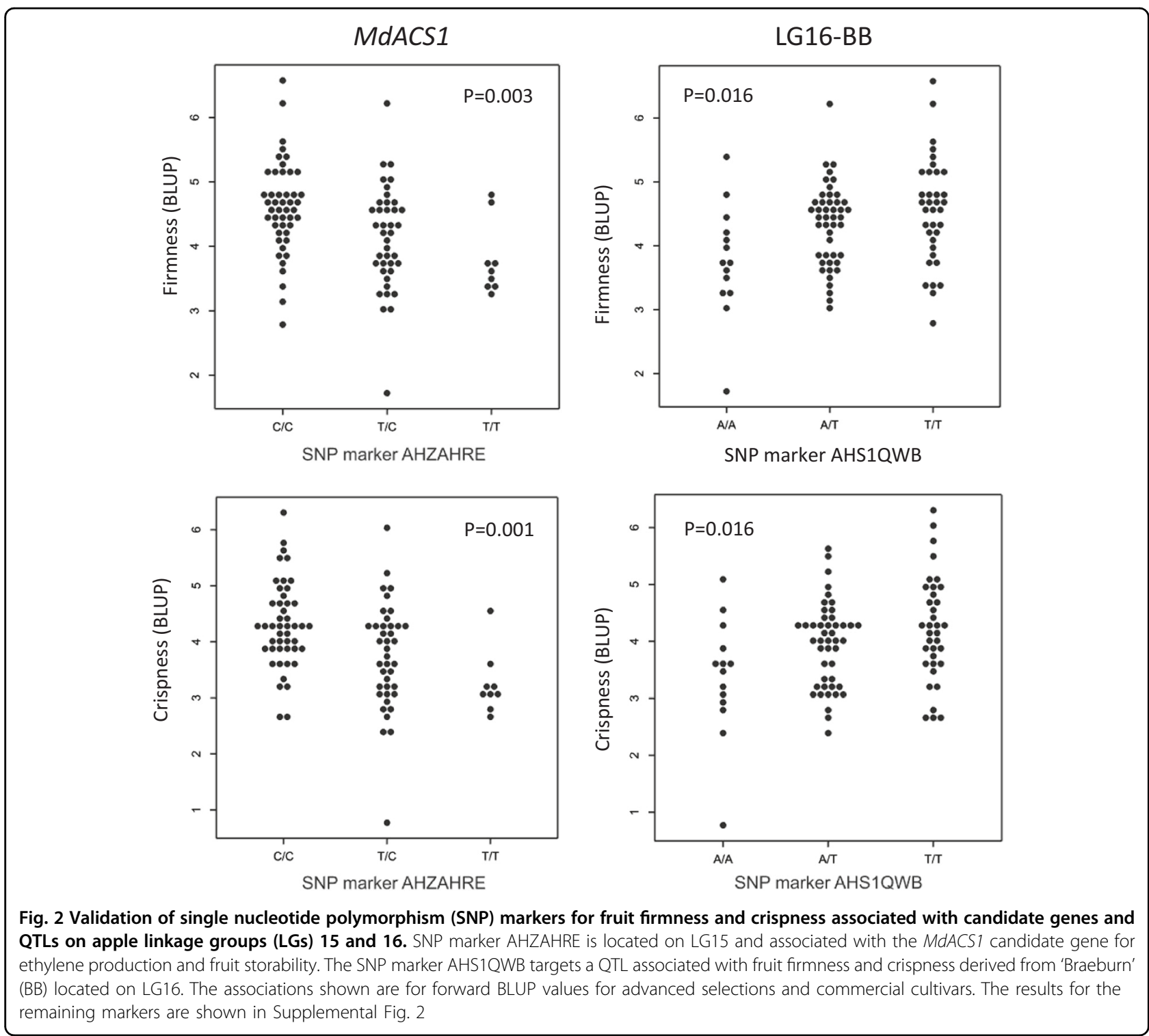

families. Markers AHHS7CA, AH6R6SR, and AHBKGG8 were significantly associated with crispness using BLUP values ( $p=0.079, p=0.058$, and $p=0.058$, respectively) but not with firmness. Conversely, AHMSZ06 was significantly associated with firmness but not with crispness, with the $\mathrm{T}$ allele being the favourable allele based on BLUPs but the unfavourable allele based on raw phenotypes. Markers AHPAWDM, AH89247, and AHABIA0 were significantly associated with firmness using the averaged phenotypes in the validation families, but not with crispness. Marker AHGJ852 was not associated with crispness nor firmness.

\section{Marker validation for fruit colour}

Of the 11 SNPs at the MYB10 candidate gene on LG9 that regulates "type 1 " red colouration in apple ${ }^{46}$,
10 amplified polymorphic markers and nine of these (AHHS7CC, AHI15IK, AHKA3OS, AHLJ1U0, AHMSZ08, AHN1X7G, AHPAWDO, AHQJUJW, and AHRSSP4) were significantly associated $(p<0.0001)$ with the proportion of overcolour on the fruit skin (Fig. 3 and Supplemental Fig. 3).

None of the six SNPs targeting the MYB110 candidate gene for "type 2" red flesh in apple amplified markers cosegregating with red flesh.

\section{Marker validation for fruit taste and aroma}

In total, 65 individuals from the validation families had phenotypic data for aroma, sweetness, acidity, and flavour intensity breeders' scores, which contributed to calculations of the marker-trait associations for fruit taste and aroma (Fig. 3; Supplemental Figure 4). 


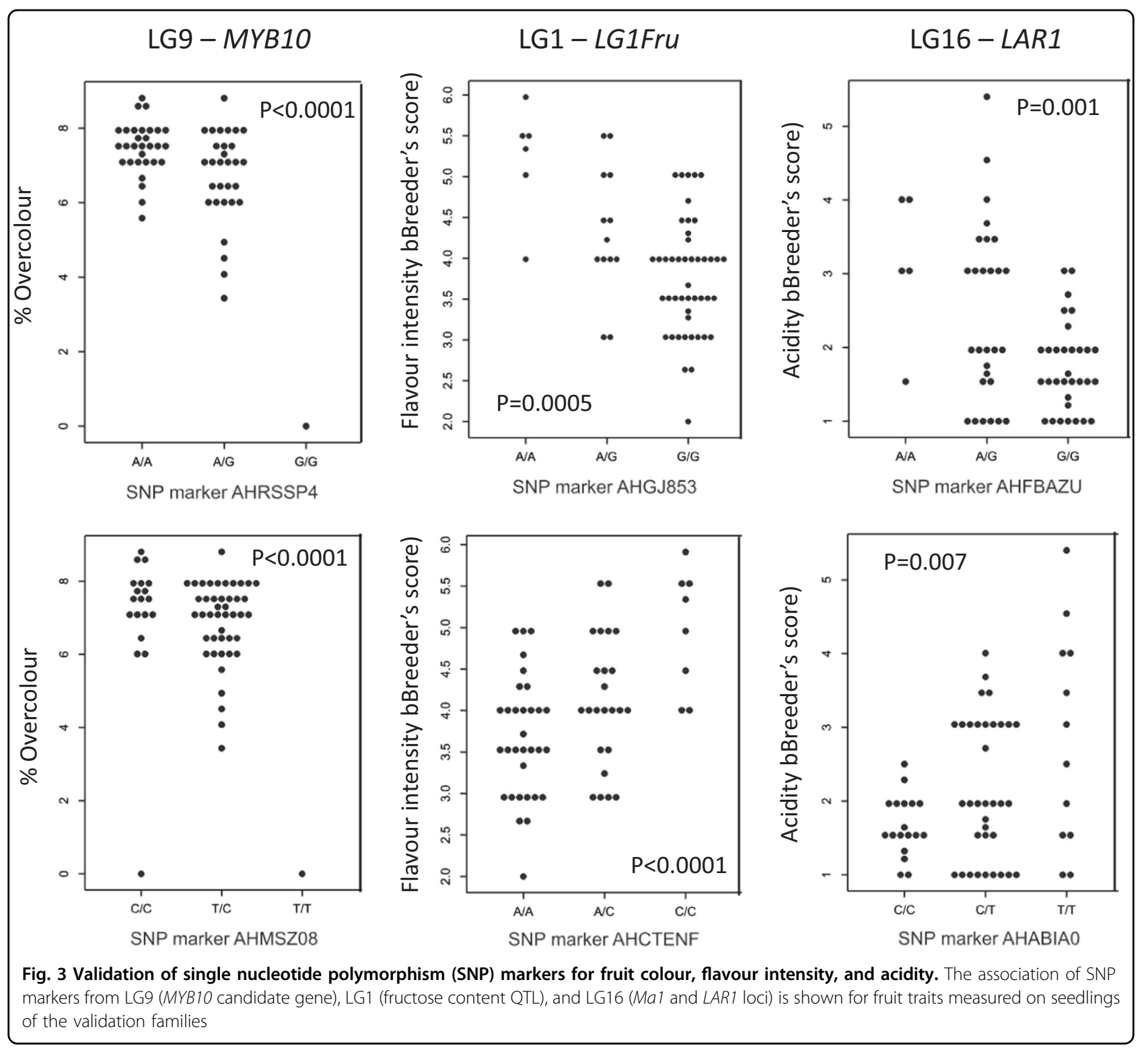

Of 25 SNPs designed for fruit acidity on LG8 and LG16, four markers on LG16 (AHFBAZU, AHHS7CA, AH89247, and AHBAIAO) were significantly associated $(p<0.01)$ with the phenotypic data of acidity in the validation families. The most highly associated marker was AHFBAZU ( $p=0.001)$, for which 26 out of 32 individuals carrying the GG genotype exhibited low fruit acidity (breeder's score $\leq 2$ ) while the AA genotype group included four out of five individuals with high acidity (breeder's score $\geq 3)$. None of the putative LG8 markers was significantly associated with acidity.

All six assays targeting the $M d A A T 1$ candidate gene on LG2 amplified polymorphic products. However, none was significantly associated with the aroma breeder's score and only one marker was weakly associated with flavour intensity (AHUAO2I, $p=0.046$; Supplemental Figure 4). Twenty-one of 27 individuals with the AA genotype for AHUAO2I were associated with a flavour intensity score $\geq 4$, while 24 of 34 AC genotypes had a score $\leq 4$.

The six assays targeting a QTL associated with fructose composition in apple were polymorphic. Although none individually was associated with the sweetness breeder's score, three markers (AHCTENF, AHFBAZV, and AHGJ853) were significantly $(p<0.001)$ associated with flavour intensity. Eighteen of 25 individuals with the AA genotype for AHCTENF had a flavour intensity score lower than 4, while all six individuals with a CC genotype had a score greater than 4 and the AC genotypes were more equally distributed (seven with low flavour intensity and 10 and high). 


\section{Marker validation for fruit phytochemical composition and disorders}

Of the 15 assays included in the IRSCOA v1.0 array targeting trait loci associated with phytochemical fruit composition such as polyphenol and vitamin C concentration, all were polymorphic; however, the PFR accessions used to genotype these markers had not been phenotyped for these compounds. Similarly, although one marker from LG13 and all the markers linked to LAR1 on LG16 had been chosen to target bitter pit disorder, it was not possible to validate the trait associations of these markers.

\section{Discussion}

\section{Evaluation of qPCR assays and IRSCOA v1.0}

In this study, we evaluated the OpenArray ${ }^{\circledR}$ technology to validate a set of SNPs that had been previously associated with phenotypic variation in important traits of apple that are commonly major targets of breeding programmes internationally. The traits were chosen to be associated with Mendelian trait loci and QTLs of varying degrees of explained phenotypic and genotypic variance. A set of robust validated markers were provided that can be used for high-throughput MAS. These validated markers will be crucial tools in the modern breeders' toolbox ${ }^{7,9}$.

We chose the OpenArray ${ }^{\circledR}$ technology because qPCR is inexpensive and $\mathrm{qPCR}$ equipment is readily available in many labs. Furthermore, qPCR chemistries are mostly transferrable among brands of qPCR machines, qPCR cycling is fast, and scoring can be semi-automated, reducing errors and enabling the integration of such assays in automated high-throughput MAS pipelines. The OpenArray $^{\circledR}$ technnology enables multiplexing of more than one marker per assay in the same experiment. In this study, 128 SNPs were included in the IRSCOA v1.0 array and $85.9 \%$ of the assays designed successfully amplified polymorphic products, indicating that the OpenArray ${ }^{\circledR}$ technnology can be readily applied for transferring variants detected by SNP arrays and re-sequencing experiments into qPCR lab-based assays. Individual qPCR assays similarly can be re-designed from the flanking sequences given in Supplemental Table 1. As an example, assay AHMSZ08 was designed using a different qPCR design and chemistry ${ }^{26}$. Other methods can be used for qPCR SNP genotyping-such as re-designing Kompetitive Allele Specific PCR (KASP; LGC, Teddington, UK), that was recently used for SNP marker development in apple ${ }^{18}$.

\section{Marker validation for pest and disease resistance}

Thirteen of 42 SNP assays from the IRSCOA v1.0 targeting pest and disease resistance loci were validated. These 13 SNP assays can hence can be implemented for MAS for the scab resistances Rvi2/Vh2, Rvi4/Vh4, Rvi6/
$V f$, fire blight resistance $M R 5 / R L P 1$, and powdery mildew resistance $P l 2$. We recommend using the AHI15IL and AHLJ1U1 assays for screening the Rvi2/Vh2 and Rvi4/ Vh4 loci, respectively, as both markers were strongly associated with resistance/susceptibility using Russian apple R12740-7 $\mathrm{A}^{41}$ as a control for resistant heterozygous genotypes and 'Gala' as a negative control for homozygous susceptible. The AHI15IL assay for Rvi2/Vh2, derived from SNP Rvi2-7_W242 described by Jansch et al. ${ }^{33}$, and is a new SNP assay not reported by Baumgartner et al. ${ }^{18}$ The AHLJ1U1 assay for apple scab resistance $R v i 4 / V h 4$ targets the same SNP as the FBsnRvi4-1_K146 assay reported by Baumgartner et al. ${ }^{18}$, providing even stronger evidence of this SNP's robustness for MAS. For the Rvi6/Vf scab resistance locus, we recommend using independently any of the four successful assays AHX1JK8, AHVJM8S, AHWSLE0, or AHZAHRG, with 'Prima' as the control for resistant heterozygous genotypes and 'Gala' as a negative control for homozygous susceptible. Although the DNA sample from 'Blenheim Orange', a cultivar susceptible to apple scab, exhibited a resistance genotype for these $R v i 6 / V f$ markers, that result might be an incorrect genotype call because this DNA sample had a somewhat low call rate (90\%) for the IRSCOA v1.0 in general. Furthermore, errors in pedigree records cannot be excluded as an explanation. Three Rvi6/Vf assays (AHVJM8S, AHWSLE0, and AHX1JK8), independently predictive, are new alternatives to the MS8_124 KASP assay developed by Baumgartner et al. ${ }^{18}$ that corresponds to the AHZAHRG assay, which is the assay we recommend as it has also been validated in the aforementioned study. For the fire blight resistance locus segregating from $M$. × robusta 'Robusta 5 ' located on $\mathrm{LG}^{32}$, we recommend using any of the AH0JFXM, AH21B92, AH4AAGA, AH5I8MI, or AH6R6SQ assays independently, with 'Robusta 5' as a control for resistant heterozygous genotypes and 'Gala' as a negative control for homozygous susceptible. For the $\mathrm{Pl} 2$ resistance locus, we recommend using either the AHD2CTO or AHFBAZW assay with $M$. $z u m i$ as a control for resistant heterozygous genotypes and 'Gala' as a negative control for homozygous susceptible. The observation that one seedling individual was homozygous G/G for AHD2CTO and heterozygous for AHFBAZW deserves closer attention, which may be due to the presence of a null allele for AHD2CTO. No markers were validated among the genotyping-by-sequencing derived SNPs from $R v i 3 / V h 3$ and from the $R v i 11 / V b j$ locus. Further efforts are required to develop robust single SNP markers for these loci.

\section{Marker validation for fruit quality traits Fruit texture}

For the purpose of MAS for firmness and crispness, we recommend four SNPs: one SNP on LG15 (AHZAHRE 
targeting MdACS1) and either of three SNPs targeting MdPG1 on LG10, AHKA3OR, AHPAWDN, and AHMSZ07. Of the 28 polymorphic assays on the IRSCOA v1.0 developed for loci associated with fruit firmness and crispness, only these four markers were significantly associated with the BLUP values for both traits of the advanced selections and commercial cultivars and then further validated using average phenotypes in advanced selection and validation families. The AHZAHRE SNP assay was designed from sequence close to the MdACS1 candidate gene that is involved in ethylene biosynthesis ${ }^{47}$ and has been associated with fruit firmness QTLs ${ }^{48}$. The same SNP was screened by Baumgartner et al. ${ }^{18}$ and our study verified that the $\mathrm{C}$ allele is associated with firmer apples. Similarly, the AHKA3OR assay was designed close to the MdPG1 candidate gene that is involved in cell wall softening $^{49}$ and has been associated with fruit firmness $\mathrm{QTLs}^{25,29,34,48}$. The same SNP (Md-PG1-SNP1) was screened by Baumgartner et al. ${ }^{18}$ and our study verified that the $\mathrm{C}$ allele is associated with firmer apples. The similar performance of the AHKA3OR, AHPAWDN, and AHMSZ07 markers may indicate that these loci are in high linkage disequilibrium in the set of samples used for this study. The single SNP tested that was associated with the $M d A C O 1$ candidate gene, although being the same as the $M d-A C O 1-S N P 1$ assay developed by Baumgartner et al. ${ }^{18}$, did not exhibit any association with fruit firmness in this study. The markers developed for the LG16 QTL detected from a 'Braeburn' family [LG16-BB ${ }^{25}$ ] were inconsistently associated with fruit firmness, indicating that further work is required to develop single markers for this locus. Alternatively, deriving haplotypes from multiple SNP assays might be needed to explain the phenotypic variation associated with this QTL, to which the successfully amplifying IRSCOA v1.0 could be applied.

The remaining 24 assays that were polymorphic showed some discrepancies between the different set of individuals and between backward BLUP and average phenotype values. These may have been caused by non-genetic factors particular to each set influencing trait values, the genetic composition of each set (for example the validation families had half the number of founders compared to the advanced selections and commercial cultivars) and non-additive genetic effects which would not be captured using backward BLUPs ${ }^{34}$.

\section{Fruit colour, acidity, aroma, and flavour intensity}

In total, nine SNPs were independently associated with fruit skin colouration, providing eight more markers around the MYB10 candidate gene in addition to the validated marker described in Chagné et al. ${ }^{26}$ Of the four markers targeting the Ma1 locus of LG16 associated with fruit acidity, we recommend using AHFBAZU, as it was the most strongly associated with the phenotype. Interestingly, the functional marker for the mutation causing a truncated malate transporter gene [Ma1-SNP1455 ${ }^{20}$, which was assay AHCTENE in our study] was not found to be the marker most associated with fruit acidity in the present study. None of the LG8 assays we designed amplified markers that were associated individually with fruit acidity. Most of these LG8 SNPs were chosen for their contribution to distinguishing several haplotypes at this locus, and therefore none of them were expected to be independently associated with fruit acidity. This difference between single SNPs and multiple SNPs at a locus explaining trait variation [addressed in Evans and Peace ${ }^{8}$ ] probably explains the observed individual SNP results for many of the trait loci targeted by the IRSCOA v1.0 assay. A single marker designed close to the AAT1 candidate gene for fruit aroma ${ }^{31,38,50}$ was weakly associated with flavour intensity; further research is required to develop a SNP assay targeting the LG2 ester composition QTL for this trait. Three markers designed on LG1 for a QTL associated with fructose composition ${ }^{39}$ were associated with flavour intensity and we recommend validating these markers in more germplasm to ascertain whether this association is real.

\section{Conclusion}

The research presented here demonstrates the successful validation of 33 apple SNP markers for 11 targeted loci using the IRSCOA v1.0 array. Each of these SNPs were independently and individually associated significantly with disease resistance or fruit quality traits. As long as a user is confident that the same associations exist in parents of a programme's breeding material, these SNPs can be used routinely for MAS for scab resistances of Rvi2/Vh2 and Rvi4/Vh4 on apple LG2, Rvi6/Vf on LG1, fire blight resistance from $M R 5 / R L P 1$ on LG3, powdery mildew resistance from $P l 2$ on LG11, fruit firmness and crispness from LG15 and LG10 trait loci including those associated with the $M d A C S 1$ and MdPG1 genes, fruit skin colouration associated with the MYB10 gene of LG9, fruit acidity associated with the Ma1 locus of LG16, and fruit flavour intensity associated with a fructose content QTL on LG1 and the MdAAT1 gene of LG2. This set of validated markers increases the range of trait-diagnostic genetic tools available for apple breeders. These markers and targeted loci are not exhaustive and more markers associated with breeding-relevant traits will be added to future assays. In addition, further utility of the IRSCOA v1.0 array might be achieved with a multi-SNP haplotype approach for some of the targeted loci.

\section{Acknowledgements}

This work was enabled by the European Commission's 7th Framework Programme FruitBreedomics Project No 265582; USDA's National Institute of 
Food and Agriculture Specialty Crop Research Initiative project "RosBREED: Combining Disease Resistance and Horticultural Quality in New Rosaceous Cultivars" (2014-51181-22378); and New Zealand's Ministry of Business, Innovation and Employment, as well as PREVAR Ltd Pipfruit Research Consortium 2 projects. We thank John Roniotis and Steve Dodds from Thermo Fisher Scientific for their help with the OpenArray ${ }^{\circledR}$ screening, and Drs Andrea Patocchi, Fabrizio Costa, Jay Norelli, Michela Troggio, Lara Poles, Daniel Sargent, Sudharsan Padmarasu, and Riccardo Velasco for contributing SNPs to the array.

\section{Author details}

${ }^{1}$ The New Zealand Institute for Plant \& Food Research Ltd (Plant \& Food Research), Palmerston North Research Centre, Palmerston North, New Zealand. ${ }^{2}$ Department of Horticulture, Washington State University, Pullman, WA, USA. ${ }^{3}$ Plant \& Food Research, Hawke's Bay Research Centre, Havelock North, New Zealand. ${ }^{4}$ USDA-ARS, National Clonal Germplasm Repository, Corvallis, OR, USA

\section{Conflict of interest}

The authors declare no conflict of interest.

\section{Publisher's note}

Springer Nature remains neutral with regard to jurisdictional claims in published maps and institutional affiliations.

Supplementary Information accompanies this paper at (https://doi.org/ 10.1038/s41438-018-0114-2).

Received: 10 May 2018 Revised: 1 November 2018 Accepted: 12 December 2018

Published online: 01 March 2019

\section{References}

1. Velasco, R. et al. The genome of the domesticated apple (Malus $\times$ domestica Borkh.). Nat. Genet. 42, 833 (2010).

2. Chagné, D. et al. The draft genome sequence of European pear (Pyrus communis L. 'Bartlett'). PLOS ONE 9, 12 (2014).

3. Verde, I. et al. The high-quality draft genome of peach (Prunus persica) identifies unique patterns of genetic diversity, domestication and genome evolution. Nat. Genet. 45, 487-494 (2013).

4. $\mathrm{Wu}, \mathrm{G}$. A. et al. Sequencing of diverse mandarin, pummelo and orange genomes reveals complex history of admixture during citrus domestication. Nat. Biotechnol. 32, 656-662 (2014).

5. $\mathrm{Wu}$, J. et al. The genome of the pear (Pyrus bretschneideri Rehd.). Genome Res. 23, 396-408 (2013).

6. Jung, S. et al. The Genome Database for Rosaceae (GDR): year 10 update. Nucleic Acids Res. 42, D1237-D1244 (2014).

7. Peace, C. P. DNA-informed breeding of rosaceous crops: promises, progress and prospects. Hortic. Res. 4, 17006 (2017)

8. Evans, K. \& Peace, C. Advances in marker-assisted breeding of apples. in Achieving sustainable cultivation of apples (ed. K. Evans) 165-194 (Burleigh Dodds, Cambridge, UK, 2017).

9. van Nocker, S. \& Gardiner, S. E. Breeding better cultivars, faster: applications of new technologies for the rapid deployment of superior horticultural tree crops. Hortic. Res. 1, 14022 (2014). https://doi.org/10.1038/hortres.2014.2

10. Kumar, S., Bink, M., Volz, R. K., Bus, V. G. M. \& Chagné, D. Towards genomic selection in apple (Malus $\times$ domestica Borkh.) breeding programmes: prospects, challenges and strategies. Tree Genet. Genomes 8, 1-14 (2012).

11. Kumar, S. et al. Genomic selection for fruit quality traits in apple (Malus $x$ domestica Borkh.). PLoS ONE 7, e36674 (2012).

12. Muranty, H. et al. Accuracy and responses of genomic selection on key traits in apple breeding. Hortic. Res. 2, 12 (2015).

13. Bernard, P. S. \& Wittwer, C. T. Real-time PCR technology for cancer diagnostics. Clin. Chem. 48, 1178-1185 (2002).

14. Edwards, S. G., O'Callaghan, J. \& Dobson, A. D. W. PCR-based detection and quantification of mycotoxigenic fungi. Mycol. Res. 106, 1005-1025 (2002).
15. Semagn, K., Babu, R., Hearne, S. \& Olsen, M. Single nucleotide polymorphism genotyping using Kompetitive Allele Specific PCR (KASP): overview of the technology and its application in crop improvement. Mol. Breed. 33, 1-14 (2014).

16. Bassett, $H$. et al. Marker assisted selection in an apple rootstock breeding family. in lii International Symposium on Molecular Markers in Horticulture (ed. R. Velasco) 25-28 (2015)

17. Bus, V. et al. Progress in pipfruit resistance breeding and research at Plant \& Food Research in XIV EUCARPIA Symp. on Fruit Breeding and Genetics (ed. S. Tartarini) (Acta Horticulturae, Bologna, Italy, 2017).

18. Baumgartner, I. O. et al. Development of SNP-based assays for disease resistance and fruit quality traits in apple (Malus $\times$ domestica Borkh.) and validation in breeding pilot studies. Tree Genet. Genomes 12, 35 (2016).

19. Vanderzande, S. et al. Crossing the finish line: how to develop diagnostic DNA tests as breeding tools after QTLdiscovery. J. Hortic. 5, 6 (2018).

20. Bai, Y. et al. A natural mutation-led truncation in one of the two aluminum-activated malate transporter-like genes at the Ma locus is associated with low fruit acidity in apple. Mol. Genet. Genom. 287 663-678 (2012).

21. Bastiaanse, $\mathrm{H}$. et al. Scab resistance in 'Geneva' apple is conditioned by a resistance gene cluster with complex genetic control. Mol. Plant Pathol. 17 159-172 (2016). https://doi.org/10.1111/mpp.12269

22. Bianco, L. et al. Development and Validation of a $20 \mathrm{~K}$ Single Nucleotide Polymorphism (SNP) Whole Genome Genotyping Array for Apple (Malus $\times$ domestica Borkh). PLoS ONE 9, e110377 (2014).

23. Bus, V. G. M. et al. Genome mapping of an apple scab, a powdery mildew and a woolly apple aphid resistance gene from open-pollinated Mildew Immune Selection. Tree Genet. Genomes 6, 477-487 (2010).

24. Chagné, D. et al. Genome-wide SNP detection, validation, and development of an 8K SNP array for apple. PLOS ONE 7, e31745 (2012).

25. Chagné, D. et al. Genetic and environmental control of fruit maturation, dry matter and firmness in apple (Malus $\times$ domestica Borkh.). Hortic. Res. 1, 14046 (2014).

26. Chagné, D., et al. A functional genetic marker for apple red skin coloration across different environments. Tree Genet. Genomes 12, 67 (2016).

27. Chagné, D. et al. QTL and candidate gene mapping for polyphenolic composition in apple fruit. BMC Plant Biol. 12, 12 (2012). https://doi.org/10.1186/ 1471-2229-12-12

28. Chagné, D. et al. An ancient duplication of apple MYB transcription factors is responsible for novel red fruit-flesh phenotypes. Plant Physiol. 161, 225-239 (2013).

29. Costa, F. et al. QTL dynamics for fruit firmness and softening around an ethylene-dependent polygalacturonase gene in apple (Malus $\times$ domestica Borkh.). J. Exp. Bot. 61, 3029-3039 (2010).

30. Costa, F. et al. Role of the genes Md-ACO1 and Md-ACS1 in ethylene production and shelf life of apple (Malus domestica Borkh). Euphytica 141, 181-190 (2005).

31. Dunemann, F. et al. Functional allelic diversity of the apple alcohol acyltransferase gene MdAAT1 associated with fruit ester volatile contents in apple cultivars. Mol. Breed. 29, 609-625 (2012).

32. Gardiner, S. E. et al. Putative resistance gene markers associated with quantitative trait loci for fire blight resistance in Malus 'Robusta 5' accessions. BMC Genet. 13, 25 (2012).

33. Jansch, M. et al. Identification of SNPs linked to eight apple disease resistance loci. Mol. Breed. 35, 45 (2015).

34. Kumar, S. et al. Novel genomic approaches unravel genetic architecture of complex traits in apple. BMC Genom. 14, 393 (2013).

35. Kumar, S., et al. Genome-wide scans reveal genetic architecture of apple flavour volatiles. Mol. Breed. 35, 118 (2015).

36. Mellidou, I., Chagné, D., Laing, W. A., Keulemans, J. \& Davey, M. W. Allelic variation in paralogs of GDP-L-Galactose Phosphorylase is a major determinant of Vitamin C concentrations in apple fruit. Plant Physiol. 160, 1613-1629 (2012).

37. Padmarasu, S. et al. Fine-mapping of the apple scab resistance locus Rvi12 (Vb) derived from 'Hansen's baccata \#2'. Mol. Breed. 34, 2119-2129 (2014).

38. Souleyre, E. J. F. et al. The AAT1 locus is critical for the biosynthesis of esters contributing to "ripe apple' flavour in "Royal Gala' and "Granny Smith' apples. Plant J. 78, 903-915 (2014).

39. Guan, Y. Z., Peace, C., Rudell, D., Verma, S. \& Evans, K. QTLs detected for individual sugars and soluble solids content in apple. Mol. Breed. 35, 13 (2015). 
40. Verma, S. et al. Two large-effect QTLs, Ma and Ma3, determine genetic potential for acidity in apple fruit: Breeding insights from a multi-family study. Tree Genet. Genomes.

41. Bus, V. G. M. et al. The Vh2 and Vh4 scab resistance genes in two differential hosts derived from Russian apple R12740-7A map to the same linkage group of apple. Mol. Breed. 15, 103-116 (2005).

42. Falconer, D. S. \& MacKay, T. F. C. Introduction to Quantitative Genetics. (Longman, Harlow, England, 1996).

43. Lynch, M. \& Walsh, B. Genetics and Analysis of Quantitative Traits. (Sinauer Associates, Sunderland, England, 1998).

44. Team, R. D. C. The R project for statistical computing (Version 2.13.0). in R Foundation for Statistical Computing, Vienna, Austria (2011).

45. Butler, D. asreml: asreml0 fits the linear mixed model. (ed. R.p.v. 3.00) (2009).

46. Lin-Wang, K. et al. High temperature reduces apple fruit colour via modulation of the anthocyanin regulatory complex. Plant Cell Environ. 34, 1176-1190 (2011).
47. Adams, D. O. \& Yang, S. F. Ethylene biosynthesis - identification OF 1aminocyclopropane-1-carboxylic acid as an intermediate in the conversion of methionine to ethylene. Proc. Natl Acad. Sci. USA 76, 170-174 (1979).

48. Costa, F. et al. Advances in QTL mapping for ethylene production in apple (Malus $\times$ domestica Borkh.). Postharvest Biol. Technol. 87, 126-132 (2014).

49. Atkinson, R. G., Schroder, R., Hallett, I. C., Cohen, D. \& MacRae, E. A. Overexpression of polygalacturonase in transgenic apple trees leads to a range of novel phenotypes involving changes in cell adhesion. Plant Physiol. 129 122-133 (2002).

50. Rowan, D. D. et al. Profiling fruit volatiles in the progeny of a 'Royal Gala' $x$ 'Granny Smith' apple (Malus $\times$ domestica) cross. J. Agric. Food Chem. 57, 7953-7961 (2009). 